\title{
Views of professional nurses regarding low tuberculosis cure rate in Greater Giyani Municipality, Limpopo Province
}

\begin{tabular}{|c|c|}
\hline \multicolumn{2}{|c|}{$\begin{array}{l}\text { Authors: } \\
\text { Nandzumuni V. Mas } \\
\text { Rachel T. Lebese }^{1} \\
\text { Lunic B. Khoza }^{1} \\
\text { Ntsieni S. Mashau }{ }^{1}\end{array}$} \\
\hline $\begin{array}{l}\text { Affiliations: } \\
{ }^{1} \text { Department } \\
\text { Nursing, Univ } \\
\text { South Africa }\end{array}$ & $\begin{array}{l}\text { ersity of Venda, } \\
\text { ersanced }\end{array}$ \\
\hline $\begin{array}{l}\text { Corresponde } \\
\text { Rachel Lebes }\end{array}$ & ce to: \\
\hline $\begin{array}{l}\text { Email: } \\
\text { rachel.lebese }\end{array}$ & @univen.ac.za \\
\hline $\begin{array}{l}\text { Postal addres } \\
\text { Private Bag X } \\
\text { Thohoyandou } \\
\text { South Africa }\end{array}$ & $\begin{array}{l}\text { 050, } \\
0950\end{array}$ \\
\hline $\begin{array}{l}\text { Dates: } \\
\text { Received: } 23 \\
\text { Accepted: } 03 \\
\text { Published: } 21\end{array}$ & $\begin{array}{l}\text { an. } 2013 \\
\text { Sept. } 2014 \\
\text { Nov. } 2014\end{array}$ \\
\hline $\begin{array}{l}\text { How to cite } t \\
\text { Maswangany } \\
\text { Lebese, R.T., } \\
\text { Mashau, N.S. } \\
\text { of profession } \\
\text { regarding low } \\
\text { cure rate in G } \\
\text { Municipality, } \\
\text { Province', Cur } \\
\text { Art. \#1148, } 8 \\
\text { dx.doi.org/10 } \\
\text { curationis.v37 }\end{array}$ & $\begin{array}{l}\text { lis article: } \\
\text { N.V., } \\
\text { hoza, L.B. \& } \\
\text { 2014, 'Views } \\
\text { I nurses } \\
\text { tuberculosis } \\
\text { eater Giyani } \\
\text { impopo } \\
\text { ationis 37(1), } \\
\text { oages. http:// } \\
4102 / \\
\text { i1.1148 }\end{array}$ \\
\hline $\begin{array}{l}\text { Copyright: } \\
\text { (C) 2014. The } \\
\text { Licensee: AOS } \\
\text { OpenJournals } \\
\text { licensed unde } \\
\text { Commons Att } \\
\text { License. }\end{array}$ & $\begin{array}{l}\text { IS } \\
\text { IS } \\
\text { This work is } \\
r \text { the Creative } \\
\text { ribution }\end{array}$ \\
\hline Read online: & \\
\hline 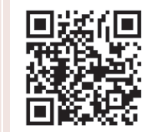 & $\begin{array}{l}\text { Scan this QR } \\
\text { code with your } \\
\text { smart phone or } \\
\text { mobile device } \\
\text { to read online. }\end{array}$ \\
\hline
\end{tabular}

Authors:

aswanganyi ${ }^{1}$

Affiliations:

${ }^{1}$ Department of Advanced

Nursing, University of Venda,

Correspondence to

Email:

Postal address:

Private Bag X5050

Thohoyandou 0950

South Africa

Accepted: 03 Sept. 2014

to cite this article:

Lebese, R.T., Khoza, L.B. \&

Mashau, N.S., 2014, 'Views

cure rate in Greater Giyan

Municipality, Limpopo

Art. \#1148, 8 pages. http://

dx.doi.org/10.4102/

Copyright:

(C) 2014. The Authors

icensee: AOSIS

licensed under the Creative

Commons Attribution
Background: Management of patients suffering from tuberculosis (TB) after discharge from hospital plays a critical role in the cure rate of TB. Despite interventions developed by the World Health Organization (WHO) to improve the cure rate, TB remains a worldwide health problem.

Objective: The purpose of the study was to explore and describe the views of professional nurses regarding the low TB cure rate in primary healthcare facilities of Greater Giyani Municipality in Limpopo Province, South Africa, with the aim of determining strategies that can be used to improve this low rate.

Method: This study was qualitative, exploratory, descriptive and contextual in nature. The population consisted of professional nurses working in primary healthcare facilities within Greater Giyani Municipality, which has a TB cure rate below the national target of $85 \%$. Data gathering was through individual face-to-face interviews using an interview guide. Open-coding was used to analyse the data in this study.

Results: The theme that emerged from data was 'factors contributing to low TB cure rate'. This theme was supported by the following sub-themes: poor referral system, lack of knowledge about TB and its treatment, stigma attached to TB, and cultural and religious beliefs. The professional nurses suggested counselling of TB patients upon diagnosis, advice about patients' responsibilities and the involvement of family members.

Conclusion: The involvement of community stakeholders in TB prevention, health promotion and education activities devoted to disease spread and cure is vital so that the stigma attached to TB can be eliminated. TB education and awareness programmes should be included in the curriculum of primary schools.

\section{Introduction and background}

Tuberculosis (TB) remains a worldwide healthcare problem, although it can be cured within 6-8 months of taking antituberculosis treatment. The most unresolved challenge in the management of TB is the lack of treatment completion, which manifests in high death rates and the occurrence of drug-resistant TB (World Health Organization [WHO] 2009a:31). In 2009 the cure rate of TB was at $78 \%$ globally and the rate of treatment success was only reached by three regions, namely the Eastern Mediterranean at 85\%, the Western Pacific at 92\%, and South-East Asia at $87 \%$ (WHO 2009b:21). In the African and American regions the treatment success rate was $75 \%$ and $76 \%$ respectively. South Africa managed to reach a $73 \%$ TB treatment success rate, which is far below the global average treatment success rate of 85\% (WHO 2009b:20).

In order to improve the cure rate globally, the Stop TB strategy was adopted in 2006, aimed at:

- pursuing high-quality directly observed treatment short course (DOTS);

- expansion and enhancement, addressing TB and/or HIV, multidrug-resistant TB (MDR-TB) and the needs of poor and vulnerable populations;

- contributing to health system strengthening based on primary health care (PHC);

- engaging all care providers; and

- empowering people with TB and communities through partnership and promoting research.

This global plan to stop TB also indicates how and at what scale the strategy should be implemented (WHO 2009a:6), as well as outlining the major interventions that should be implemented to achieve goal number six of the Millennium Development Goals, which aims to combat HIV and/or AIDS, malaria and other diseases. The goal states that countries should begin to reverse the incidence of TB by 2015 and to reduce the prevalence and mortality rates by 50\% 
relative to 1990 levels (Dye et al. 2006:461). Other targets are to achieve a case detection rate of new smear-positive cases of at least $70 \%$ and to reach a treatment success rate of $85 \%$ (Dye et al. 2006:461).

The Stop TB strategy implementation rate is not yet at $100 \%$; only 18 countries are implementing it. Progress against TB has been made between 1995 and 2005 as a result of the implementation of DOTS and Stop TB. It was reported that amongst patients notified with TB in 2008, $85 \%$ were successfully treated, reaching the $85 \%$ target of successfully treated new smear-positive cases (WHO 2009b:37). Globally DOTS coverage had reached $94 \%$ in 2007, which does not correlate with the cure rate of less than $70 \%$. All WHOdefined regions have adopted the DOTS strategy, but they differ in coverage as follows: Africa 93\%; America 91\%; Mediterranean 97\%; Europe 75\%; South-East Asia 100\%; and Western Pacific 100\% (WHO 2009b:34). The percentage of DOT coverage does not support the low cure rate still being experienced in the different countries, especially in Africa.

According to the WHO the implementation of DOTS in South Africa by 2009 was 100\% (WHO 2009a:35). However, in the Limpopo Province District Health System report, DOTS coverage in 2007 was ranging between $75 \%$ and $80 \%$. DOTS coverage in Mopani District was $94 \%$. According to a report from the District Health Information System (Gariep et al. 2008:547), Greater Giyani Municipality DOTS coverage ranges between $90 \%$ and $96 \%$, but the cure rate of $70.2 \%$ is still lagging behind the national target TB cure rate of $85 \%$.

In many developing countries such as South Africa less than half of the TB patients who started treatment were cured or completed the treatment course (WHO 2009b:38). In 2008 Limpopo Province had a TB cure rate of $65 \%$ and a defaulter rate of 7\%; in Mopani District the TB cure rate was $68.4 \%$ and in Greater Giyani Municipality it was 70.2\% - far below the national target of 85\% (Gariep et al. 2008:547).

\section{Problem statement}

The Greater Giyani Municipality TB cure rate for 2008 was $70.2 \%$, which is far below the national target of $85 \%$. This is of great concern from a public health perspective. Nurses who care for TB patients have never been interviewed as to their opinions concerning the reasons why the TB cure rate did not reach the national target. Despite the measures that are in place to assist in increasing of TB cure rate, such as human and financial resources as well as availability of TB guidelines in all facilities, the TB cure rate in Mopani District was still below the national target of 85\% in 2008 (District Health Information System 2008:18).

\section{Aim of the study}

The purpose of the study was to explore and describe the views of professional nurses regarding the low TB cure rate in PHC facilities of the Greater Giyani Municipality in the Mopani District of Limpopo Province. The research objectives were to explore how TB patients are managed after discharge from hospital; and to determine strategies that can be used to improve the low TB cure rate within Greater Giyani Municipality.

\section{Definition of key concepts}

Professional nurse: A person who is qualified and competent to independently practise comprehensive nursing in the manner and to the level prescribed, and who is capable of assuming responsibility and accountability for such practice (South African Nursing Council 2005:6). In this study a professional nurse is a nurse who is taking care of people infected with and affected by TB in the selected PHC facilities of Greater Giyani Municipality, with low TB cure rates.

Low TB cure rate: In this study a low TB cure rate is defined as the percentage of cured patients (who have been diagnosed through sputum examination) that is below the national target of $85 \%$.

Primary healthcare facilities: These are facilities which are accessible to individuals and the services are offered at an affordable cost (Dennil, King \& Swanepoel 2007:2). In this study PHC facilities are clinics and health centres in Greater Giyani Municipality which render TB prevention and treatment programmes.

\section{Significance of the study}

The results of the study may assist in contributing to development of strategies to improve the low TB cure rates within Greater Giyani Municipality. Challenges associated with low TB cure rates will be revealed and recommendations for improvement will be made. It is hoped that the research will inform policy makers so that they will consider factors that can improve TB cure rates. The results of this study might assist in contributing to the upgrading of educational programmes for nurses and in schools.

\section{Research method and design}

A qualitative, explorative, descriptive and contextual design was used to explore the views of professional nurses regarding the low TB cure rate in Greater Giyani Municipality. Professional nurses narrated their views during a face-to-face interview guided by an interview guide. The conversation with participants was recorded and field notes were used to document nonverbal cues. Participants were interviewed in their own settings, which were PHC facilities. Probing and paraphrasing were used to elicit more information and clarity from the participants.

The findings of this study were later documented and direct quotes from the interviews and literature control were used to support them. The data were used to produce a descriptive account; this was reviewed by three selected participants, who confirmed their accuracy (Burns \& Grove 2005:361; Creswell 2003:190; Polit \& Beck 2004:718; Neuman 2011:38). 


\section{Population}

Population is defined as the total number of units from which data can potentiality be collected (Polit \& Beck 2007:28). The population was made up of all professional nurses who were taking care of TB patients in facilities with a low TB cure rate in Greater Giyani Municipality. Facilities were sampled based on the low percentage TB cure rate. Professional nurses were selected purposively because of their knowledge of working with TB patients (Polit \& Beck 2007:28). The sample comprised of 18 professional nurses, who were all females. Their ages ranged between 31 and 48 years. All had more than two years' experience of working with TB patients.

\section{Data collection}

Data gathering was through an individual face-to-face interview using an interview guide (Creswell 2003:181). The researcher asked participants questions to stimulate their thoughts. Probing and follow-up questions were asked according to the participants' responses. The main question that directed the interview guide was: 'In your view what do you think could be the causes of the low TB cure rate in the Greater Giyani Municipality of Limpopo Province?' The duration of individual interviews depended on how the participant responded to probing questions. In-depth individual interviews were conducted and data saturation occurred after 18 professional nurses were interviewed.

\section{Data analysis}

The researcher used qualitative data analysis methods and Tech's open-coding method to guide the researcher in this study (De Vos 2005:350). All data were read carefully, with understanding, to obtain a sense of the whole. Ideas were written down as they came to mind. The researcher selected one transcript from one interview and asked what this was about and thought about the underlying meaning in the information. The researcher's thoughts were then written in the margin. A list was made of all themes and similar themes were clustered together. The researcher applied the list of themes to the data. The themes, abbreviated as codes, were then written next to the appropriate segments of the transcripts. Themes and sub-themes were developed (De Vos 2005:350-361).

\section{Ethical considerations}

Ethical considerations were taken into account throughout the study. Permission to conduct the study was sought from the following institutions after ethical clearance was obtained from the University of Venda Health and Safety Research Ethics Committee: Limpopo Province Department of Health and Social Development Research Ethics Committee and Mopani District of Limpopo Province.

The researcher did not allow unauthorised persons to have access to the raw data of the study. Information was not attached to names of participants or institutions.
Records were kept locked away at all times and the personal computer that was used had a password to allow access to the researcher only (Burns \& Grove 2005:196). Findings were not linked to the names of participants, who are known to the researcher only. Their names will not be used in documents.

Informed consent means that participants have adequate information regarding the research, comprehend the information and have the power of free choice (Polit \& Beck 2007:93). This would enable them to consent voluntarily to participate in the research or decline participation. Participants were allowed to make informed decisions regarding participation in this study. The researcher explained the purpose of the study and the procedures involved, potential risks and/or benefits, how confidentiality would be maintained and the right to withdraw from participation. The researcher ensured that the explanation was at the level of the participants' comprehension. The participants then voluntarily gave consent, which was in written form (Burns \& Grove 2005:196).

Privacy was maintained throughout the process of data collection and interviews were conducted in a private room marked 'do not disturb' as a measure to ensure the privacy of participants. Permission to use the voice recorder was sought from participants and they were informed of the right to refuse and stop the recording. The participants were also shown the stop button on the audiotape so that they could press the button if they felt that the information they wanted to disclose should not be recorded (Burns \& Grove 2005:196).

The researcher ensured that participants were comfortable by selecting a convenient venue, time and day. The researcher also guarded against embarrassing the participants during interviews. Questioning and probing was therefore done cautiously. During the interview the researcher was vigilant in assessing the participants' discomfort and made provision for referral to professional intervention if needed (Burns \& Grove 2005:196).

\section{Trustworthiness}

Four principles were applied to ensure trustworthiness: credibility, dependability, conformability and transferability (Brink 2006:118). Activities to ensure credibility included member checking and prolonged engagement by going back to the participants for data verification; reflexivity and peer review by engaging supervisors and peers in seminar presentation; and use of different timeframes for interviews. The strategy of transferability was used to ensure applicability in other contexts by a dense description of the research process and findings. The strategy of dependability was used to ensure consistency, which was enhanced by coding and recoding of data by an independent coder with experience in qualitative research. The strategy of confirmability was used to ensure neutrality. Transcripts and audiotapes were made available to supervisors to confirm the findings. 


\section{Results and discussion}

In-depth individual interviews were conducted and data saturation occurred after 18 professional nurses had been interviewed. One theme and sub-themes were developed, as summarised in Table 1.

\section{Factors contributing to low TB cure rate}

During data collection in this study professional nurses expressed their views regarding the low $\mathrm{TB}$ cure rate in Greater Giyani Municipality. One theme emerged: factors contributing to low TB cure rate. The following sub-themes were identified from this theme: poor referral system, lack of knowledge about TB and its treatment, stigma attached to $\mathrm{TB}$, and cultural and religious beliefs.

\section{Poor referral system}

During interviews professional nurses expressed different factors that they believe contributes to the low TB cure rate. A poor referral system between the hospital and PHC was identified as one of the factors contributing to the low TB cure rate. In this study professional nurses described the referral system as being poor because patients are given referral forms from the hospital and told to go to the PHC facility with that referral form, without a proper explanation of its importance. Participants explained that the hospitals are failing to inform the PHC facilities about the discharged TB patients. This often results in patients not reaching the facilities which they are referred to. It was mentioned that there is also a lack of follow-up by the referring facility, and patients are discovered by chance during outreach activities or feedback on performance done by the TB coordinator. As one participant said:

'We only become aware that there is a patient who was supposed to come to our facility after 2-3 months when the local TB coordinator has given us a print-out of the report of the patients transferred from the hospital to the primary health care facilities, and by that time the patient has already defaulted from treatment.' (Participant 15)

In a study conducted by Edington and Hodkinson (2006:1019) it was revealed that poor referral of TB patients from Chris Hani Baragwanath Hospital to PHC facilities resulted in poor TB outcomes which included low TB cure rates due to patients who were lost to treatment. A study conducted by Wandwalo et al. (2004:112) revealed that patients were also lost between public and private healthcare institutions and programmes of TB and HIV due to poor collaboration. Another study by Dong et al. (2007:492) revealed that discharged patients were instructed to go to a TB office located near the front gate of the hospital for completion of discharge procedures; those

\section{TABLE 1: Summary of findings.}

\begin{tabular}{ll}
\hline Theme & Sub-themes \\
\hline Factors contributing to low & Poor referral system. \\
TB cure rate. & Lack of knowledge about TB and its treatment. \\
& Stigma attached to TB. \\
& Cultural and religious beliefs. \\
\hline
\end{tabular}

TB, tuberculosis. who bypassed the TB office were missed, and in the end defaulted from taking treatment. From what was described it is clear that a poor referral system can affect the TB cure rate because there is poor communication between the hospital and the PHC facilities.

\section{Lack of knowledge about TB}

Data in this study revealed that there was a lack of knowledge about TB as a disease amongst TB patients. Professional nurses in this study revealed that patients seemed not to be aware of the dangers of not completing TB treatment and as a result moved from one place to another without notifying the healthcare professionals. According to the Health Belief Model (HBM) people will not change their behaviour unless they believe that they are at risk (Akinsola 2006:68). Participants in this study explained that TB patients move from one farm to another without continuing with TB treatment and therefore risk the complications associated with TB:

'TB patients go to farms without letting us know about their movement, we only get the report when we visit their families that they are working on the farms. The challenge is that they don't stay on one farm, they move from one farm to the other and this is difficult to trace. This movement affects their management and collection of treatment from the primary health care facility'. (Participant 4)

It was further revealed in this study that when feeling better some patients visit relatives without informing the nurses, which results in treatment default.

The HBM revealed that change in behaviour to avoid the consequences of disease depends on how serious people consider the consequences to be (Akinsola 2006:68). This study showed that TB patients do not take the consequences of the disease seriously, which led to complications and death. Participants in this study further indicated that delay in seeking treatment was caused by lack of knowledge about the signs and symptoms of TB. They also said that TB patients default from treatment when they feel better, because they think that they are cured.

The literature indicates that delay in diagnosis and initiation of treatment contributes to low TB cure rates of below the national target of $85 \%$ (Kiwuwa, Karamagi \& Mayanja 2005:122). It was also found that smokers delay in seeking medical assistance because they associate coughing with their smoking and not with TB symptoms (Kiwuwa et al. 2005:122). It was also revealed that alcoholics and subsistence farmers also delayed visiting health facilities due to lack of knowledge about signs and symptoms (Kiwuwa et al. 2005:122).

Lack of an opportunity to express their feelings amongst people suffering from $\mathrm{TB}$ is said to have contributed to defaulting amongst TB patients. However, Elbireer et al. (2011:985) revealed that lack of relationships with patients affects adherence, because patients do not have trust in the nurses and will not comply with the instructions given to 
them since they do not even trust that the treatment is the correct one and will be able to cure their disease (Elbireer et al. 2011:985). The HBM indicates that people are not easily convinced about changing their behaviour unless they know that they will benefit as a result of that change (Akinsola 2006:68).

Another study by Thiam et al. (2009:383) revealed that there was no proper provision of information between patients and healthcare providers on diagnosis and registration and during continuity of treatment. The same study indicated that patients were not given enough information about $\mathrm{TB}$, and this resulted in them defaulting from taking treatment. A study by Muhammad et al. (2011:1490) indicated that there was inequality in the knowledge of TB between urban and rural residents. The same study revealed poor knowledge of signs and symptoms, transmission, prevention and treatment of $\mathrm{TB}$ in people from rural areas.

In their study also conducted in Mopani District Mabunda and Bradley (2011:99) showed that lack of information about TB and its treatment had a negative impact on the management of TB patients. TB patients who experienced side-effects dropped out of treatment after two months of effective treatment and lied about being discharged. In the same study by Mabunda and Bradley (2011:99) participants indicated that TB patients who claimed to be adhering to treatment were throwing away some of the treatment, believing that they are lessening the side-effects within their bodies. It was noted from the discussion with professional nurses in this study that TB patients lack the necessary information about their condition, which resulted in lack of adherence to treatment.

\section{Stigma attached to TB}

Participants described how stigma attached to TB impacted negatively on the management of TB patients. It is indicated in the HBM that people are reluctant to change their behaviour because of fear of having difficulties during that process of change (Akinsola 2006:69). In this study participants explained that stigma can prevent the patient from taking TB treatment because it is difficult to visit the facility for assistance when you know that the disease has stigma attached to it. In this study participants expressed that stigma attached to TB acted as a barrier to patients seeking medical assistance and adhering to TB treatment.

Professional nurses identified factors which showed that TB patients were affected by the stigma attached to the condition. Participants in this study further explained that patients tend to use facilities far from their homes due to stigma attached to having TB.

During interviews with the professional nurses it was revealed that the stigma attached to TB had an impact on its management. The use of facilities far from the TB patients' homes was identified as the main factor which affects management of the TB patients, since it impacts on supervision of treatment. Professional nurses said that they were unable to attach patients to the treatment supporters, and the patients took responsibility for taking treatment alone. Participants in this study also indicated that it was very difficult for them to follow up on those patients because they sometimes switched their cell phones off.

Participants in this study expressed concern that TB patients were not disclosing their TB status to family members because of fear of isolation by family members and neighbours. Data also revealed that people suffering from TB who were working did not disclose their TB status to their employers because of fear of expulsion from work. This may result in a negative impact on the management of TB patients, because the patient will take treatment without supervision. Professional nurses in this study were concerned that if TB is treated with secrecy, treatment adherence can be affected.

It was also revealed that when patients come from far, professional nurses have difficulties in attaching TB patients to DOTS supporters:

'We have difficulty in the management of patients who are coming from the villages far from our facility because we don't know the treatment supporters in their villages. We just give the person treatment for self-supervision and tell them how to take the tablets on a daily basis. Sometimes we try to ask them to bring a person that they feel can support them with medicine administration, but they normally don't bring them.' (Participant 10)

A study conducted by Shiluvane et al. (2011:131) in Vhembe District of Limpopo Province on perceptions of people about TB revealed that people had misconceptions about TB symptoms, transmission, causes and risk factors. It was also mentioned that these misconceptions resulted in neighbours and the families of TB patients not sharing anything with them because of fear of contracting the disease (Shiluvane et al. 2011:131). A study by Tang and Squire (2005:99) indicated that people diagnosed with TB were afraid of being known about in the community because they were afraid of being stigmatised. Those who were working were afraid of losing their jobs when their employers discovered that they have TB. It was also said that TB patients do not want even their families to know about their TB status, especially the women, because they were afraid that their husbands would divorce them as they feared that their children would be infected (Tang \& Squire 2005:99).

It was further reported that families also isolate the TB patient because they are also afraid of societal isolation (Kelly 2010:238). According to Juniarti and Evans (2011:1966) TB was viewed as a bad and dirty disease by the community and the patients were isolated both by their families and the community.

In a study conducted in Vhembe District by Sukumani et al. (2011:166) it was revealed that TB patients experience a feeling of social isolation due to the stigma attached to the relationship between TB and HIV infection. It was also 
revealed that neighbours and relatives do not want to visit the families of those patients because they fear contracting HIV infection. Shiluvane et al. (2011:131) revealed that people who contracted TB were thought to be dirty and to eat bad food, and were looked down upon by others and stigmatised. The findings of this study indicated that TB patients do not want their TB status to be known due to fear of stigma about the disease.

According to the HBM people will change their behaviour only if they see that they will benefit from it. In this situation, where disclosure of the disease will bring negative effects, there will be no treatment supporter and non-adherence will be the result.

\section{Cultural and religious beliefs}

During interviews with professional nurses it was revealed that people suffering from TB were affected by their religious beliefs. They described visits to traditional health practitioners and faith healers as common practice amongst TB patients. Data indicated that cultural beliefs about TB strengthen the issue of stigma, which contributes to non-adherence to treatment and complications of the disease. Participants in this study explained that TB patients take TB treatment together with traditional medicine and end up not being cured. Participants have noted that TB patients develop drug resistance and others die due to failure to adhere to treatment. Participants in this study indicated that they thought that the reason why people suffering from TB prefer using traditional medicine was because they associate the disease with witchcraft. Participants were also concerned that people suffering from TB who are discharged from the hospitals are lost to treatment because sometimes they consult traditional or faith healers who give them alternative treatment.

It was revealed in this study that some of the TB patients believed in God and faith healers and that God will decide as to what comes next in their lives. This may affect TB patients in that they have faith only in God and do not have trust in TB treatment to cure them. Nothing encourages them to take the treatment. In the HBM it was said that the faith that a person has in the ability to do something has an impact on the actual ability to do it. In this situation it is clear that the TB patients do not have any faith in TB treatment, and nothing positive can be done to make them take treatment.

\section{Participant 7 said:}

'TB patients get wrong messages from the traditional health practitioners and faith healers who make them believe that they are not suffering from TB but it is a result of witchcraft. Others believe that it is a result of sleeping with a woman whose husband had passed on before the ritual ceremony is performed. TB patients also believe that the treatment from the health facilities will not be able to cure that type of TB.'

Participant 13 stated: 'Some of the TB patients are mixing the traditional medicine and the TB drugs and those patients result in treatment failures. Others developed drug resistance and in others their conditions worsened and they died.'
The literature indicates that traditional health practitioners are powerful allies in TB and HIV programmes; separation make patients believe that they should choose between Western health treatment and traditional cultural practices. It was reported that TB patients have trust in traditional health practitioners; therefore the Western healthcare system should work together with traditional health practitioners in order to win the TB patients over (Dong et al. 2007:493). It was reported that in Somalia beliefs concerning TB infection ranged from it being punishment for dishonesty, hereditary, or due to witchcraft, overwork and loss of faith. It was also believed that the successful treatment of TB was in the hands of God because he is the one who created them, he knows what they will undergo through their lives until death, and there is no way that it can be changed by doctors or anybody else (Citrin 2006:2000). A study by Muhammad et al. (2011:1490) revealed that people were using traditional medicine and going to priests to receive prayers in order to get cured.

A study by Hoa et al. (2003:61) in Vietnam revealed that patients' beliefs have a negative impact on cure rate. In the same study it was found that patients believed that TB was caused by hard work, heredity and too much thinking. The same study also revealed that TB patients believed there was an alternative treatment to cure TB depending on what caused it - rather than using treatment from health institutions. TB patients visited traditional health practitioners and faith healers because they did not have the knowledge that the treatment provided at the health facilities was the only cure for TB (Van der Werf, Dade \& Van der Mark 2009:250). Fochsen, Deshpande and Thorson (2009:164) also indicated that religious and cultural beliefs not only delay the patient from getting cured, but also worsen the patient's condition because they may develop drug resistance, whilst others die.

According to Liongo (2003:81) there are different types of TB, the African and the Western type. The first one is believed to be caused by having sex with someone who had a spontaneous abortion, and can be treated by traditional health practitioners; the second is believed to be caused by spread from TB sufferers as a result of smoking and environmental pollution, and can be treated by TB drugs (Liongo 2003:81).

From what was described, it was noted that cultural and religious beliefs have an influence on the stigma about TB. Discussions with professional nurses revealed that cultural and religious beliefs may contribute in lowering the TB cure rate because people suffering from TB may not adhere to TB treatment because of them.

\section{Limitations of the study}

The study was conducted in one municipality of Mopani District. The study was qualitative and therefore the results cannot be generalised. However, an in-depth description of the study process is provided to ensure replicability in other contexts. 


\section{Recommendations}

Based on the findings of this study, the following recommendations are made:

- TB patients should be counselled upon diagnosis so that they understand their condition and comply with treatment. Family members should be included in the process of counselling and education, so that they can support the patient in adhering to treatment.

- There should be collaboration between all stakeholders involved in taking care of people suffering from TB and HIV and/or AIDS in the community to ensure continuity and adherence to treatment. Traditional and faith healers should be included in the continuum of care of people suffering from TB and other diseases such as HIV and/or AIDS in the community.

- Hospitals should develop strategies to improve the referral system to PHC facilities to avoid confusion which will result in TB patients discontinuing their treatment before they are cured.

- Community awareness raising about TB should be conducted to empower people with the knowledge of TB in order to minimise stigma attached to the disease.

\section{Conclusion}

In this study professional nurses expressed their views regarding the low TB cure rate in Greater Giyani Municipality. Factors contributing to the low $\mathrm{TB}$ cure rate emerged as a theme, and the following sub-themes were identified from the main theme: poor referral system, lack of knowledge about TB and treatment, stigma attached to TB, and cultural and religious beliefs. Professional nurses indicated that there was poor communication between the hospitals and the PHC facilities, which resulted in patients not adhering to treatment. The study revealed that TB patients preferred to visit PHC facilities which were far from their homes due to fear of stigma, which also resulted in defaulting on treatment.

\section{Acknowledgements}

We thank the University of Venda for funding this study, the participants for freely giving information and our co-authors for contributing to this article.

\section{Competing interests}

The authors declare that they have no financial or personal relationship(s) that may have inappropriately influenced them in writing this article.

\section{Authors' contributions}

M.N.V. (University of Venda) conducted the research, the methodology was designed by R.T.L. (University of Venda) and L.B.K. (University of Venda) provided the conceptual guidance, whilst M.N.S. (University of Venda) sourced most of the literature.

\section{References}

Akinsola, H.A, 2006, A-Z of community health in medical, nursing and health education practice, 2nd edn, College Press, Ibadan.

Brink, H.I., 2006, Fundamentals of nursing research, 5th edn., W.B. Saunders, Philadelphia.

Burns, N. \& Grove, S.K., 2005, The practice of nursing research, conduct, critique \& utilization, 4th edn., W.B Saunders, Philadelphia.

Citrin, D., 2006, 'Somali tuberculosis cultural profile', Ethnomed 1(1),1995-2012.

Creswell, J.W., 2003, Research design: Qualitative, quantitative, and mixed method approaches, Sage Publications, Thuosand Oaks, CA.

Dennil, K., King, L. \& Swanepoel, K., 2007, Aspects of primary health care, 2nd edn., Oxford University Press, Cape Town.

De Vos, A.S., 2005, Research at grass roots: A primer for a caring profession, Van Schaik, Pretoria.

District Health Information System, 2008, Mopani District TB Report, Department of Health, Limpopo Province, South Africa.

Dong, K., Thabethe, Z., Hurtado, R., Sibaya, T., Dlwati, H., Walker, B. \& Wilson, W. 2007, 'Challenges to the success of HIV and tuberculosis care and treatment in the public health sector in South Africa', Journal of Infectious Diseases 196(3) 491-496. http://dx.doi.org/10.1086/521111

Dye, C., Watt, C.J., Dolin, P., Bleed, D.M., Hosseini, S.M. \& Raviglione, M.C., 2006 'Evolution of tuberculosis control and prospects for reducing tuberculosis incidence, prevalence and death globally burden of tuberculosis,' Journal of the American Medical Association 293, 2767-2777. http://dx.doi.org/10.1001/ jama.293.22.2767

Edington M.E. \& Hodkinson, H.J., 2006, Tuberculosis at Chris Hani Baragwanath Hospital: An intervention to improve patient referrals to district clinics, International Tuberculosis \& Lung Disease 10(9), 1018-1022.

Elbireer, S., Guwabuddhe, D., Mudiope, P., Sekandi, J.N. \& Manabe Y.C., 2011, 'Tuberculosis patients in urban Uganda. Default among HIV-TB co-infected', Tropical Medicine \& International Health 16(8), 981-987. http://dx.doi. org/10.1111/j.1365-3156.2011.02800.x

Fochsen, G., Deshpande, K.C. \& Thorson, A., 2009, 'Conflicting accountabilities: Doctors' dilemma in tuberculosis control in rural India', Health Policy 89, 160-167. http://dx.doi.org/10.1016/j.healthpol.2008.05.013

Gariep, A., Stoos, N., Mckenzie, A., Dlamini, L., Govender, T., Ponde, J. \& Herbst, K., 2008, 'An evaluation of the District Health Information in rural South Africa', South African Medical Journal 98(7), 546-552.

Hoa, N.P., Thorson, A.E.K., Long, N.H. \& Diwan, V.K., 2003, 'Knowledge of tuberculosis and associated health seeking behaviour among rural Vietnamese adults with a cough for at least three weeks', Scand J Public Health Suppl 62, 59-65. http:// dx.doi.org/10.1080/14034950310015121

Juniarti, N. \& Evans, D., 2011, 'A qualitative review: The stigma of tuberculosis', Journal of Clinical Nursing 20, 13-14. http://dx.doi.org/10.1111/j.13652702.2010.03516.x

Kelly, P., 2010, 'Isolation and stigma: The experience of patients with active tuberculosis', Journal of Community Health Nursing 16(4), 233-241. http://dx.doi. org/10.1207/S15327655JCHN1604_3

Kiwuwa, M.S., Karamagi, C. \& Mayanja, K.H., 2005, 'Patient and health service delay in pulmonary tuberculosis patients attending a referral hospital: A cross-sectional study', BMC, Public Health 5(122), 1186-1471.

Liongo, I., 2003, 'Tuberculosis health beliefs: Gaps of tuberculosis and suspected tuberculosis cases in New York City', International Journal of Clinical Health Psychology 4(1), 69-90.

Mabunda, J.T. \& Bradley, H., 2011, 'Factors contributing to poor performance of Directly Observed Treatment Short-course in Mopani district of Limpopo Province, South Africa', African Journal for Physical, Health Education, Recreation and Dance 2(1), 93-107.

Muhammad, U.M., Shaid, U, Muhammad, A Saed A, Omor, F., Mushtaq, A.M Siddiqui, M. \& Kram, J.A., 2011, 'Urban-rural inequalities in knowledge, attitudes and practices regarding TB in two districts of Pakistan's Punjab province', International Journal for Equity in Health 10(8).

Neuman, W.L., 2011, Social research methods: Qualitative and quantitative approaches, 7th edn., Pearson, Boston.

Polit, D.F. \& Beck, C.T., 2004, Nursing research: Generating and assessing evidence for nursing practice, North American edition, Lippincott, Philadelphia.

Polit, D.F. \& Beck, C.T., 2007, Nursing research: Generating and assessing evidence for nursing practice, North American edition, Lippincott, Philadelphia.

Shiluvane, N.G., Risenga, P.R., Khoza, L.B. \& Lebese, R.T., 2011, 'Perceptions of people about tuberculosis in Vhembe District of Limpopo Province, South Africa', African Journal for Physical, Health Education, Recreation and Dance 2(1), 124-134.

South African Nursing Council, 2005, The scope and practice of professional nurses, South African Nursing Council, Pretoria.

Sukumani, T.J., Lebese, R.T., Khoza, L.B. \& Risenga, P.R., 2011, 'Challenges faced by family members in home-based tuberculosis care in Vhembe District of Limpopo Province, South Africa', African Journal for Physical, Health Education, Recreation and Dance 2(1), 161-171.

Tang, S. \& Squire, S.B., 2005, 'What lessons can be drawn from Tuberculosis Control in China in the 1990s. An analysis from a health system perspective', Health Policy 72, 93-104. http://dx.doi.org/10.1016/j.healthpol.2004.06.009 
Thiam, S., Lefevre, A.M., Hane, F., Ndiaye, A., Ba, F., Fielding, K.I., Ndir, M. \& Lienhardt, C., 2009, 'Identifying barriers to effective tuberculosis control in Senegal: An anthropological approach', internationatjour $5(5), 539-543$.

Van der Werf, T.S., Dade G.K. \& Van der Mark, T.W., 2009, 'Patient compliance with tuberculosis treatment in Ghana: Factors influencing adherence to therapy in a rural service programme', Tubercle 7, 247-252.
Wandwalo, E., Kapalata, N., Tarimo, E. Corrigan, C.B. \& Morkve, O., 2004, 'National Tuberculosis \& Leprosy Programme, Ministry of Health', African Health Sciences South Africa 4(2), 109-114.

World Health Organization, 2009a, Global tuberculosis control: Surveillance, planning and financing, World Health Organization, Geneva.

World Health Organization, 2009b, Global tuberculosis control: Epidemiology, strategy, financing, World Health Organization, Geneva. 